\title{
Ecología política del agua y territorialización de las luchas sociales. La experiencia del foro hídrico de Lomas de Zamora
}

\section{María Gabriela Merlinsky}

Universidad de Buenos Aires

merlinsk@retina.ar

RESUMEN

En este artículo desarrollamos un análisis de las acciones colectivas por el acceso al agua y el saneamiento en la metrópolis de Buenos Aires. Nos interesa dar cuenta de la construcción social y política de la cuestión hídrica. Para ello, analizamos la emergencia de conflictos y reclamos por justicia ambiental, donde se elaboran nuevos conocimientos acerca del ciclo hidrosocial. La investigación se basó en un estudio de caso que analiza la experiencia del «Foro Hídrico de Lomas de Zamora», una organización que desarrolla acciones en la cuenca baja del Matanza-Riachuelo. El trabajo busca explicar la resonancia politica de estas acciones en términos de la territorialización de los conflictos y la producción de conocimiento contraexperto. Nos proponemos mostrar la resonancia que tiene esta experiencia en la organización colectiva y en la construcción de nuevos lenguajes de derechos.

Palabras clave: conflictos ambientales, ecología política del agua, movimientos socioambientales, conocimiento contrahegemónico, derecho humano al agua, territorialización de los conflictos. 


\section{Political ecology of water and territorialization of social struggle. Lomas de Zamora Water Forum's experience}

\section{ABSTRACT}

This article we develops an analysis of collective action for water access and sanitation in the Buenos Aires metropolis. It intends to give an account of the social and political construction of water issue. To this end, it analyzes the emergence of conflicts and claims for environmental justice that create new knowledge about the hydrosocial cycle. The research was based on a case study that examines the experience of the "Foro Hidrico de Lomas de Zamora» (Lomas de Zamora Water Forum), an organization developing actions in the lower basin of the Matanza-Riachuelo river. The results of the study show the political resonance of these actions in terms of the territorialization of conflicts and the production of counter-expertise knowledge. We aim to show the resonance that this experience has in collective organization and the construction of new languages of rights.

Keywords: environmental conflicts, political ecology of water, social-environmental movements, counter-hegemonic knowledge, water human right, territorialization of conflicts. 


\section{INTRODUCCIÓN}

A comienzos de la década de 1980 visité por primera vez Villa Jardín, en el municipio de Lanús, un asentamiento localizado en los márgenes del Riachuelo, en la cuenca más contaminada de Argentina. La traza planificada con acceso a servicios públicos del lado de la Capital Federal se desvanecía al cruzar el puente que permitía atravesar el río en dirección a la provincia de Buenos Aires, donde uno se perdía en un laberinto de viviendas informales sin cloacas ni agua potable.

Al ingresar al barrio, se hacía visible otra imagen impactante que daba cuenta de una animosa vida comunitaria. En espacios comunes y en reducidos terrenos, los habitantes construían sus casas, apuntalaban locales comunitarios y producían bienes y servicios para diferentes mercados. Ese ir y venir cotidiano se desplegaba en un paisaje urbano muy peculiar: las viviendas, asentadas en sitios de relleno de lo que había sido una antigua laguna, tendían a hundirse y por las áreas comunes corrían los efluentes cloacales y los vertidos domésticos por canales a cielo abierto. Por aquel entonces, las organizaciones del barrio exigían a diferentes agencias estatales la provisión de servicios sociales, sin embargo, nadie planteaba que la falta de agua y cloacas fuera un problema socioambiental que causara efectos sobre la salud.

Desde entonces he tenido a cargo diferentes investigaciones en torno a las experiencias colectivas de organización en los barrios de ese territorio vasto que es la cuenca Matanza-Riachuelo. En ese recorrido, he podido constatar que, ya entrado el siglo XXI, diferentes experiencias de organización local frente a los problemas hídricos se han transformado en demandas políticas que tienen visibilidad pública. Esto se debe a la proliferación de un sinnúmero de conflictos en los que diferentes comunidades se han organizado para reclamar por el acceso a recursos esenciales para la supervivencia familiar (Merlinsky, 2007; Merlinsky, 2014; Merlinsky, 2016). 
Recurriendo a diferentes enfoques de la ecología política, la sociología ambiental, la geografía crítica y a las investigaciones que analizan los movimientos de justicia ambiental en diferentes regiones del planeta, hemos podido establecer que se trata de conflictos ambientales o ecológicos distributivos. Más precisamente, se trata de respuestas a la expansión de actividades económicas que conllevan la extracción intensiva de recursos, expansión de vertederos y riesgos de contaminación para quienes el ambiente es la base material del sustento (Guha y Martínez Alier, 1997; Martínez Alier, 2004). En ese sentido, podemos decir que - a dos décadas de mi primera visita a un asentamiento informal en los márgenes del Riachuelo - lo que ha cambiado es que los daños causados por la contaminación se han hecho visibles en las comunidades y son experimentados como tales. Cuando los afectados se sienten amenazados, dentro de su propia concepción de la vida, por riesgos que perciben como significativos, es que deciden organizarse y protestar contra el daño. Este último punto es un aspecto a ser abordado en este artículo.

En los últimos años hemos observado diferentes experiencias de movilización que cuestionan y establecen responsabilidades sobre el acaparamiento de los recursos, no solo el agua: también la tierra, la biodiversidad o la infraestructura básica, entre otros (Blanchon et al., 2009; Naguib Pellow y Brulle, 2005; Firpo, 2012). En estas experiencias, la definición del problema se construye en torno a la defensa de la vida, se establecen múltiples conexiones entre humanos y no humanos y se producen diferentes definiciones acerca de la justicia ambiental (Descola, 2005; Di Chiro, 1998; Schlosberg, 2012; Vainer, 2004; Faber, 2005).

A partir de estudiar diferentes conflictos por el acceso al agua y el saneamiento en la cuenca Matanza-Riachuelo (Merlinsky et al., 2012; Merlinsky, 2014; Merlinsky, 2016b), hemos podido constatar que un elemento catalizador de la acción colectiva es la construcción de conocimiento contraexperto. Se trata de modalidades de acción en las cuales los pobladores y organizaciones territoriales, con la ayuda de grupos expertos, desarrollan diferentes investigaciones para establecer los orígenes de un problema que los afecta. Estos relevamientos forman parte de un ejercicio de la epidemiología popular ${ }^{1}$ donde la enumeración de causas y efectos toma en consideración variables igualmente importantes, como el papel que juegan los intereses empresarios o las políticas públicas, entre

Brown define como epidemiología popular al accionar mediante el cual ciudadanos comunes (legos o no expertos) reúnen estadísticas, información, recursos y conocimiento experto para comprender la epidemiología de una enfermedad (Brown, 1987, p. 78). 
otros factores (Brown, 1987; P. Brown y Mikkelsen, 1990; Callon, Lascoumes y Barthe, 2001; Ackrich et al, 2010).

Esta asociación entre diferentes eventos socionaturales y la búsqueda de responsabilidades políticas permite un análisis crítico de los flujos de agua, el modo en que son manipulados por determinadas geometrías de poder para ser direccionados hacia determinados usos y grupos sociales (Swyngedouw, 2004, 2009; Budds y Hinojosa, 2012; Molle, 2012). Todo ello contribuye a desnaturalizar la situación de exposición al peligro tóxico y permite la construcción del problema como un asunto de derechos (Merlinsky, 2013).

Entre los casos estudiados, uno que llamó poderosamente nuestra atención fue la experiencia del «Foro Hídrico de Lomas de Zamora» ${ }^{2}$, una red que lleva adelante acciones en diferentes barrios de la cuenca Matanza Riachuelo y que tiene su núcleo fundacional en el barrio Lamadrid, en el municipio de Lomas de Zamora. Esta organización ha ido ganando capacidad de influencia pública a comienzos de este milenio, en el escenario de creciente conflictividad social posterior a los episodios de diciembre de 2001.

Este artículo desarrolla un análisis de la acción colectiva del Foro Hídrico en el marco de la lucha por el acceso al agua y el saneamiento en la metrópolis de Buenos Aires, buscando dar cuenta de la construcción social y política de la cuestión hídrica. Nos interesa mostrar de qué modo estos colectivos construyen saberes contraexpertos, al tiempo que elaboran y contraponen lenguajes de valoración alternativos al discurso del estado y las corporaciones. Dos preguntas importantes de nuestra investigación refieren a establecer cuáles han sido los procesos colectivos que permitieron que el agua se volviera un asunto de preocupación social y de qué manera se han producido transformaciones políticas en pos de la construcción de la cuestión hídrica como un asunto que merece atención estatal.

La estrategia teórico-metodológica de la investigación se ha apoyado en un estudio de caso instrumental (Stake, 1995). Se trata de un caso relevante por la permanencia de estos reclamos en el tiempo (la experiencia que ya lleva dos décadas y sus antecedentes se remontan hacia la década de 1980) y porque ha generado efectos y consecuencias en términos de un debate sobre las decisiones públicas y el derecho de los afectados a participar en ellas.

Las fuentes de datos provienen de entrevistas en profundidad a diferentes pobladores de Villa Lamadrid, y también de entrevistas grupales e individuales con los integrantes del Foro Hídrico que residen en diferentes barrios de la cuenca

\footnotetext{
2 En adelante el Foro Hídrico.
} 
Matanza-Riachuelo. Asimismo, hemos consultado a referentes de organizaciones no gubernamentales, expertos en la temática hídrica, funcionarios municipales, de la Autoridad de la Cuenca Matanza-Riachuelo y representantes de Agua y Saneamiento S.A. (AySA) y reportes de estas últimas agencias estatales.

\section{DESIGUALDAD SOCIAL Y CUESTIÓN HÍDRICA EN LA METRÓPOLIS DE BUENOS AIRES}

En Buenos Aires, el avance en la expansión de las redes de agua y saneamiento tuvo su época de oro a comienzos del siglo XX. En efecto, la creación de Obras Sanitarias de la Nación en 1912 dio inicio al período de la gran intervención federal en materia de agua y saneamiento (De Gouvello, Lentini y Brenner, 2012). Se trató de un plan de obras que logró garantizar la cobertura del servicio para la Capital Federal y que luego se extendió hacia la conurbación a finales de la década de 1930 con la creación del Área Sanitaria Metropolitana, que incluía a trece municipios, entre ellos Lomas de Zamora, el territorio de nuestro caso de estudio ${ }^{3}$.

Este sistema metropolitano luego entró en un proceso de estancamiento, lo que coincidió con un período de intenso crecimiento urbano en los bordes de la ciudad. En efecto, durante el auge del modelo de industrialización por sustitución de importaciones, importantes contingentes de población migraron, se instalaron en el territorio de la cuenca Matanza-Riachuelo, y los espacios que encontraron en la ciudad muchas veces se encontraban en terrenos inundables donde, en la mayoría de los casos, construyeron sus viviendas usando mano de obra familiar. Como esta expansión no estuvo acompañada por la provisión de servicios esenciales, la población debió recrear condiciones de «urbanidad» que el Estado no llegó a garantizar (Tobías, 2014, p. 44). Entre las décadas de 1950 y 1960 se rompe el equilibrio entre el crecimiento de la ciudad y la expansión de la red de agua y saneamiento, lo que contribuye a una diferenciación socioespacial acentuada por los diferentes grados de equidad en el acceso al servicio (Catenazzi, 2006, pp. 120-121).

A partir de entonces sobrevino un extenso período de desinversión del sector, hasta que en 1993, bajo un gobierno de corte neoliberal, se privatiza el servicio a través de su concesión a la empresa Aguas Argentinas. Fue entonces cuando

El Área Sanitaria Metropolitana abarcaba en ese momento los partidos de Almirante Brown, Avellaneda, Esteban Echeverría, La Matanza, Lanús, Lomas de Zamora, Morón, San Fernando, San Isidro, San Martín, Tres de Febrero, Tigre y Vicente López, y la Capital Federal. 
se dejó atrás el enfoque social del agua y se fue construyendo un nuevo modelo de gestión cuyo supuesto fundamental fue la comodificación del recurso hídrico (Swyngedouw et al., 2002). En el período que va desde 1993 hasta 2006, los precios de mercado definieron la posibilidad de acceder al servicio, hubo un incremento acelerado de tarifas, se detuvo la inversión y aumentaron las brechas entre grupos y clases sociales según su posibilidad de estar conectados a la red de agua y saneamiento.

En el año 2006, en medio de una crisis del sector por la falta de cumplimiento de los contratos de concesión, el servicio de agua fue reestatizado y pasó a manos de Agua y Saneamiento Sociedad Anónima (AySA), sociedad del Estado. El diagnóstico en ese momento era de un déficit de cobertura de tres millones de personas sin servicio de agua potable y seis millones con carencia de desagües cloacales. La nueva empresa diseñó un plan director con la meta de llegar al 100\% de cobertura en el año 2020. En los últimos diez años (2006-2016) hubo avances significativos en el tendido de la red de agua, pero la evolución de la cobertura fue mucho menor en relación con la extensión de la red cloacal ${ }^{4}$, entre otras cosas porque se requieren obras de envergadura que permitan ampliar la capacidad del sistema mediante grandes colectores troncales, las que son condición previa para construir nuevos conductos.

Si consideramos el área de concesión de AySA, el servicio cloacal alcanza a cubrir el total de la población de la Capital Federal, mientras que, en la zona norte, la cobertura alcanza al $72 \%$ del área servida, y en la zona sur (el área que coincide con la mayor parte de la cuenca Matanza-Riachuelo) tan solo el 59\% de la población tiene cobertura de servicios sanitarios (AySA, 2013).

Se ha analizado la vulnerabilidad sanitaria de la población de la cuenca Matanza-Riachuelo considerando la incidencia combinada del riesgo sanitario ${ }^{5}$, la densidad habitacional y el nivel socioeconómico. La población en situación

4 La cobertura de agua en el área de concesión se incrementó en poco más de dos puntos porcentuales en el período 2001-2010, es decir, del $81 \%$ a $83 \%$ de los hogares. En lo que refiere a la cobertura de servicios cloacales, si bien hubo un aumentó en 3,5 puntos porcentuales $(61 \%$ a $64 \%$ ), esto no alcanzó para lograr una expansión significativa en relación con la brecha histórica entre el abastecimiento de agua y la provisión del servicio cloacal. Esta inequidad es aún más acentuada en la región sur del área de concesión, donde la cobertura de agua pasó del $73 \%$ al $76 \%$ y la cobertura de servicios cloacales pasó del 33\% a 38\% de los hogares (Censos Nacionales de Población y Vivienda, 2001, 2010).

5 El riesgo sanitario es una variable compleja que se mide a partir de cuatro indicadores: la carencia de servicios de agua potable, la carencia de servicios de desagües cloacales, la presencia de menores de cinco años y la probabilidad de sufrir inundaciones (AySA, 2009). 
de alta vulnerabilidad sanitaria tiene una cobertura de agua potable del $40 \%$ y una cobertura media de servicios de desagües cloacales de solo el 10\%. En esas mismas áreas, la pobreza cuadriplica a la observada en las áreas de vulnerabilidad sanitaria baja, alcanzando en términos medios al $66 \%$ de la población. El resultado del análisis mostró que el 53\% de la población que habita en la cuenca se encuentra expuesto a elevados niveles de riesgo sanitario (2 004245 habitantes), de los cuales 541754 habitantes merecen especial atención por habitar áreas de alta densidad poblacional (AySA, 2009, p. 18). Esta situación es representativa de las villas y asentamientos que se ubican en el borde del Riachuelo, en el municipio de Lomas de Zamora.

\section{EL PROCESO DE OCUPACIÓN DEL SUELO Y DE CONSTRUCCIÓN DE ORGANIZACIONES TERRITORIALES EN LOMAS DE ZAMORA. LA EXPERIENCIA EN VILLA LAMADRID}

El municipio de Lomas de Zamora forma parte del primer cordón del conurbano bonaerense y tiene una población de 619279 habitantes. Esto lo convierte en el segundo partido más poblado del Gran Buenos Aires. En su interior es posible identificar dos áreas geográficas diferenciadas: las localidades ubicadas hacia el sudeste, que se encuentran en zonas altas, y las localidades ubicadas al noreste - aledañas al curso del río Matanza Riachuelo-, que se emplazan en terrenos bajos e inundables.

En la metrópolis de Buenos Aires, la construcción de viviendas ha estado comandada por operaciones mercantiles destinadas a grupos de ingresos mediosaltos y altos. En contraposición, no se han generado políticas de suelo para sectores populares que hayan tenido duración en el tiempo. Por esa razón, las ocupaciones colectivas de tierra representan uno de los principales medios para acceder al hábitat popular (Cravino, 2008; Jaramillo, 2008).

En Villa Lamadrid, los pobladores fueron construyendo sus viviendas en las proximidades de los márgenes del Matanza-Riachuelo, en terrenos muy bajos y porosos. En los testimonios de los vecinos más antiguos, las referencias a las inundaciones periódicas se remontan a las décadas de 1960 y 1970, es decir el período de conformación definitiva del barrio. Los habitantes más antiguos destacan que, en años posteriores, la situación se fue agravando por el aumento de la densidad poblacional, por falta de obras de infraestructura y porque no se cumplieron las regulaciones municipales en los usos del suelo. En efecto, el barrio se densificó 


\section{División interna del partido de Lomas de Zamora y municipios limítrofes en la cuenca baja del Matanza-Riachuelo}

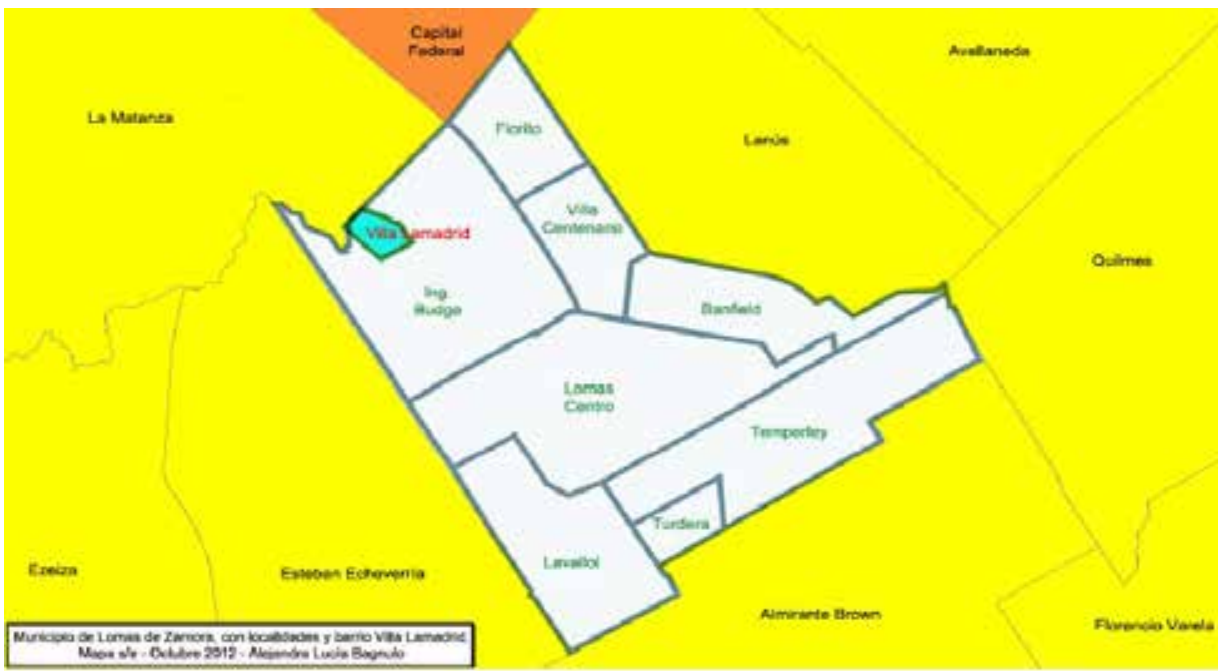

Fuente: Alejandra Bagnuolo (2012). Informe Socioambiental del barrio Lamadrid.

aún más a finales de la década de 1980, cuando la crisis económica expulsaba a los inquilinos de la Capital Federal hacia el conurbano y, para hacer frente a la crisis habitacional, los «sin techo» optaron por formar parte de ocupaciones colectivas de tierras en lotes sin servicios. Como las zonas más protegidas de las inundaciones ya estaban ocupadas, estos nuevos habitantes ocuparon áreas deprimidas cubiertas de agua, que fue necesario rellenar. Por aquella época, la conexión de red se hizo insuficiente y la principal carencia que unía los reclamos de antiguos y nuevos pobladores era la falta del servicio cloacal. Fue entonces cuando se formó la organización Agua para Todos, que exigía la construcción de un «tercer refuerzo», es decir, un gran canal troncal que permitiera conectar los barrios a la red de agua y saneamiento de la ciudad.

Como respuesta a esa demanda, la concesionaria privada Aguas Argentinas puso en marcha un plan de infraestructura para llevar el agua desde la ciudad central hacia la periferia mediante un nuevo conducto que transportaría el agua de fuente superficial extraída del Río de La Plata hacia los barrios de la cuenca baja del Matanza-Riachuelo. En este plan quedaron sin incluirse las obras de conexión cloacal y esto generó un desbalance hídrico: por ser un territorio bajo cota de inundación, las napas freáticas comenzaron a aumentar su nivel, y por mucho tiempo, la única solución fue la instalación de bombas depresoras. 
En el año 2000 se produjo una gran inundación en la zona como producto de una temporada de lluvias, pero esta vez el agua tuvo mayor dificultad en retirarse por la dificultad de absorción de los suelos. Con 20000 evacuados y declarada la emergencia hídrica en varios municipios de la cuenca baja, los vecinos de Villa Lamadrid convocaron a pobladores y organizaciones de diferentes barrios en la cuenca Matanza-Riachuelo para formar un nuevo colectivo: el Foro Hídrico. Esta inundación extraordinaria representó un punto de inflexión para la agregación de demandas colectivas, la redefinición de la escala del problema y la asignación de responsabilidades institucionales.

\section{LA INSCRIPCIÓN TERRITORIAL DE LA CUESTIÓN HÍDRICA. EL FORO HÍDRICO COMO ESPACIO DE EXPERIMENTACIÓN SOCIAL}

Cuando consultamos a los participantes sobre el origen del Foro Hídrico, la respuesta suele venir acompañada con una referencia a la actividad de «lanzar la alerta» (Bernstein y Jasper, 1998), que es característica en los movimientos que reclaman por afectaciones ambientales. Se trata de la apertura de una temporalidad marcada por procesos de asociación, de cooperación, de comunicación y movilización pública. Buscando llamar la atención sobre un problema común, se extienden los marcos injusticia hacia un conjunto organizacional más amplio (Cefai, 2007, p. 576), se genera cierta influencia en la opinión pública y se producen reacomodamientos políticos en torno a los marcos de acción colectiva (Snow y otros, 1986). Como señala Alfredo, uno de los activos participantes en el Foro Hídrico:

La organización se hizo mucho más fuerte, pero además amplió la visión que fuimos teniendo del problema, y como se rechazaban las causas que nosotros planteábamos [fundamentalmente por Aguas Argentinas], como ellos no se hacían cargo, eso nos llevaba a buscar las razones. Y sobre todo, buscar aliados, porque estamos hablando del tránsito del gobierno de Menem a la Alianza; por esa época, muchos políticos participaban por esa razón. Y llegó en aquel entonces, en el año 2000, la gran inundación, que fue un golpe fuertísimo. Es allí cuando decidimos, en julio del 2000, tener una herramienta que se llamaría Foro Hídrico. Además, tomábamos nuestros recaudos con los partidos políticos para lograr apoyos de diferentes agrupaciones. Nosotros ampliábamos la petición, no nos centrábamos en el problema de las napas, decíamos que la solución era mucho más amplia. Fuimos dándonos cuenta de que para solucionar el problema hídrico en la zona se necesita tener un proyecto integral (entrevista a Alfredo, Foro Hídrico, 27-09-2006). 
Pero no solo la inundación extraordinaria produjo esta conexión entre organizaciones. Lo que también dio impulso al proceso colectivo fue un contexto político particular que tuvo lugar ya avanzado el año 2001 con la emergencia de una de las crisis económicas más graves de la historia argentina, cuando un colapso del aparato productivo, bancario y de las finanzas públicas condujo a un estallido social y a innumerables protestas sociales que acabaron finalmente expulsando al presidente de la Nación. Por entonces, las clases medias movilizadas y los sectores populares encontraron en las asambleas barriales la posibilidad de redefinirse identitariamente desde la acción política y construir lazos de solidaridad entre diferentes sectores sociales (Svampa, 2008). Esto permitió que se recrearan formas políticas prexistentes, que emergieran nuevos actores sociales y que antiguos problemas adquirieran nueva fisonomía.

Desde el retorno de la democracia - e incluso mucho antes, durante la última dictadura militar-, diferentes líderes barriales de Villa Lamadrid, Cuartel Noveno e Ingeniero Budge habían participado en experiencias comunes, tanto en comités de inundados como en agrupaciones políticas y diferentes grupos multisectoriales. Como parte de estas experiencias, reclamaban por la suba de tarifas, la construcción de escuelas, la realización de infraestructuras, el acceso al agua potable, la tenencia de la tierra y la gestión del riesgo de inundaciones, entre otras tantas demandas. Muchas veces se habían encontrado en diferentes acciones de protesta en acampes, cortes de ruta, festivales y campañas de prensa. A finales del año 2001 y durante el año siguiente, cuando en diferentes lugares de Argentina se produce la expansión del fenómeno asambleario, son estos vecinos los que se acercan y redoblan su participación en las reuniones del Foro Hídrico para debatir, compartir conocimientos y hacer un monitoreo de formas y tiempos de protesta.

Fue entonces cuando las deliberaciones tuvieron como epicentro el territorio de la cuenca Matanza-Riachuelo. Si bien, como hemos visto, ya existían conexiones entre actores diversos, nunca antes se había producido una inscripción territorial de los reclamos en torno a un encuadre regional. A partir de ese momento, el Riachuelo comenzó a ser nombrado como un espacio social cargado de valor y las aguas del río se volvieron objeto de resignificación como paisaje y en términos identitarios (Melé, 2007; Trom, 1999, p. 36). La cuenca baja del Matanza-Riachuelo se volvió para estas organizaciones un espacio de resistencia y un lugar de creación de nuevas relaciones sociales. La definición de ese territorio como un lugar afectado implica no solo dar cuenta de los cuerpos dañados sino también establecer nuevas conexiones entre la naturaleza y la vida comunitaria. 
Lo que pasa es que vivimos en una cuenca, vivir en una cuenca significa haberle robado terreno al río y después nosotros lo que hacemos es combatir al río. La cuenca es terreno aledaño [...], es la naturaleza que le da al río cierto lugar determinado, para cuando hay ciertos excesos de la naturaleza y no puede sostener los caudales, los va derivando en ese lecho que es propiedad del río. La naturaleza es muy sabia en ese sentido [...]. Si nosotros hemos atropellado y somos intrusos en un lugar determinado, tenemos que tomar las medidas para compaginar la existencia de ambos, del ser humano con el río. El río tiene tanto derecho como nosotros. El problema generalmente son las medidas que se toman, que son medidas en contra del río [...]. Levantamos acá una cosa para que no pueda venir, y el río tiene más fuerza, en algún lugar va a encontrar su lugar para seguir su camino, la naturaleza sigue su camino, la vida sigue su camino. Entonces nosotros lo que tenemos que hacer son proyectos de convivir en una zona, quien se le ocurrió que acá en la zona baja tendría que haber casas de otro tipo, parecidas a las que hay allá donde están todas las islas del tigre o algo (entrevista a Juan, Foro Hídrico, 24-04-2005).

Esta inscripción territorial del problema hídrico también aparece en los testimonios de Walter, quien valora el conocimiento de los cambios que se van produciendo en el territorio como la fuente más importante para identificar los problemas. En sus palabras, se trata de encarar una lucha integral.

Si a la falta de cloacas le sumas el cambio en el régimen de lluvia y la falta de absorción de la tierra, todo eso hace que el desborde de los arroyos lleve el agua con el contenido de los pozos ciegos a tu casa de vuelta, asentándose en la tierra, las paredes, los colchones, creando una situación sanitaria cada vez peor [...]. Entonces, no podés decir «estamos por las cloacas, pero no nos afligimos por las napas o por las inundaciones», porque es el mismo problema. Por eso nosotros determinamos que nuestra lucha es una lucha integral en este territorio que es la cuenca Matanza-Riachuelo (entrevista a Walter, Foro Hídrico, 26-05-2009).

Una diferencia importante entre la experiencia de las asambleas de clase media en Capital Federal y la trayectoria del Foro Hídrico es que, a diferencia de aquellas, esta organización ha mantenido su presencia y capacidad de influencia pública en los últimos años. Las razones de esta singular permanencia hay que buscarlas en dos procesos importantes. El primero tiene que ver con el formato organizacional lábil de foro, el que se podría asimilar a la definición de Callon, Lascoumes y Barthe (2001) sobre los foros híbridos.

Los foros híbridos son espacios públicos de experimentación colectiva, en los que se ponen en discusión diferentes afectaciones ambientales. Son híbridos 
porque participan en ellos actores heterogéneos (pobladores, organizaciones, funcionarios, políticos, expertos, empresas de servicios, etc.) y porque los problemas que allí se discuten se inscriben en diferentes registros, que pueden ser técnicos, económicos, jurídicos, políticos, sociales, etc. Estos diferentes contenidos permiten extender la discusión y ampliar los argumentos, tomando en cuenta una pluralidad de puntos de vista, demandas y posibles respuestas. Se trata de dispositivos de exploración y de aprendizaje colectivo, porque allí se analizan los problemas, se buscan las pruebas y se define quiénes pueden ser los actores acreditados para generar el conocimiento que aportará nuevas soluciones (Callon et al., 2001, pp. 47-50). Esto permite incorporar nuevos actores y públicos heterogéneos a medida que se va complejizando la definición del problema y se extiende la escala de influencia de la organización.

La segunda razón tiene que ver con la progresiva construcción de la cuestión hídrica como un asunto de derechos, aspecto que puede traducirse en tres grandes reclamos: el derecho humano al agua, el derecho a la información y el derecho a la participación en las decisiones de política pública que tienen consecuencias en la vida comunitaria. Como veremos en los próximos apartados, se trata de acciones colectivas que tienen la capacidad de enlazar actores heterogéneos, que permiten construir la demanda como un asunto de reclamo a los poderes públicos y que evocan los repertorios de los movimientos de justicia ambiental en diferentes lugares del mundo.

\section{EL DERECHO HUMANO AL AGUA. EL ELEMENTO CATALIZADOR EN LA DEFINICIÓN DE LA CUESTIÓN HÍDRICA}

El Comité de Derechos Económicos, Sociales y Culturales de las Naciones Unidas estableció, en su observación $\mathrm{N}^{\circ} 15$, que el agua es un derecho humano fundamental, lo que implica su reconocimiento como derecho universal, inalienable e inviolable. En ese mismo marco, y el de otras directivas internacionales, el acceso al agua está vinculado con el derecho a la salud (artículo 12 del Pacto Internacional de Derechos Económicos, Sociales y Culturales de las Naciones Unidad) y el derecho a un nivel de vida adecuado. Todas las directivas han contribuido a instalar, al menos en el plano formal, la idea de que el acceso al agua y las cloacas son derechos universales, lo que implica la exigibilidad de la cobertura universal, independientemente de condiciones económicas particulares (Tobías, 2016).

En los últimos años, las narrativas y repertorios de acción de los grupos y activistas que forman parte del Foro Hídrico se concentran en desarrollar campañas 
para hacer exigible el derecho y lograr que se concrete en acciones efectivas y afirmativas de las políticas públicas. Esto tiene que ver con la exigencia de políticas de reconocimiento, es decir, por un igual tratamiento ante la ley y en relación con la implementación de las políticas públicas.

Desde la perspectiva de estos actores, ha sido la discontinuidad en materia de obras públicas la que, al compás de diferentes oleadas de privatización y restatización, ha dejado como saldo una brecha histórica inequitativa en la cobertura de servicios. Hemos visto que, en el período 2006-2016 hubo avances significativos en el tendido de la red de agua, pero la evolución de la cobertura fue mucho menor en relación con la extensión de la red cloacal, entre otras cosas porque ello depende de obras de envergadura, las que dependen de créditos internacionales.

Las organizaciones de Villa Lamadrid han realizado campañas para mostrar que esa brecha histórica en la cobertura del servicio afecta principalmente a los sectores populares que viven en las márgenes del Riachuelo, porque ha primado un criterio de rentabilidad en la lógica de provisión del servicio. Es más rápido y seguro recobrar la inversión cuando se trata de provisión de agua potable y, en cambio, se requieren grandes erogaciones para garantizar las obras de envergadura necesarias para expandir la red cloacal.

A partir del año 2012 el Foro Hídrico, junto al Departamento de Hidráulica de la Facultad de Ingeniería (UBA) y la Fundación Che Pibe, encabezaron otra campaña titulada «El agua no es confort, el agua es vida», buscando mostrar diferentes caras de la desigualdad territorial en el acceso a los servicios básicos: mientras algunos vecinos poseen la red de agua de AySA, otros se abastecen a través de redes vecinales, perforaciones ( algunos reciben agua a través de camiones cisterna o conexiones clandestinas.

La falta de cloacas en las zonas de mayor riesgo sanitario no es una casualidad. No es que la gente no tenga cloacas porque no tiene ganas, sino que siempre son los pobres los que han sido relegados por el negocio inmobiliario, por las empresas. Por ejemplo, cuando el servicio de OSN fue privatizado se priorizó dar servicio a gente que pueda asegurar el pago de la tarifa, y no a gente que no pueda pagarlo [...]. Sin embargo, a la larga, los costos de no hacer las obras públicas los está pagando el Estado a través de la salud (entrevista a Alfredo, Foro Hídrico, 14-04-2013).

Para los integrantes del Foro Hídrico, que vieron aumentadas sus expectativas con la noticia de la restatización del servicio, esta demora en la implementación del Plan Director de AySA es una constatación de que hay barreras políticas en términos de la definición de prioridades de obras. Las organizaciones cuestionan 
un modelo de racionalidad técnica que requiere inversiones de largo plazo, las que implican la realización de importantes obras de infraestructura para hacer los colectores cloacales (construcción de un nuevo colector en el margen izquierdo del Riachuelo) como prerrequisito para extender el servicio. Esto implica un tiempo de espera de quince años para poder acceder a las cloacas.

El argumento de los integrantes del Foro Hídrico cuestiona este modelo de análisis de costo-beneficio, planteando que la satisfacción de las necesidades de la población que está en riesgo sanitario no puede ser postergada. En esta construcción de argumentos, el problema se inscribe públicamente como un reclamo al Estado por la discontinuidad en las obras de infraestructura y por la falta de criterios de justicia ambiental que puedan compensar las inequidades en el acceso a los recursos.

[En referencia a las grandes obras del Plan Director] Es un proyecto muy a largo plazo. Y va a depender no solo de la acción política de este gobierno, sino de los gobiernos futuros. Estamos hablando de que acá a quince años esas obras terminen. Yo no sé si realmente lo que dicen en quince años se termina, viene otro gobierno y dice: «No, espera, hay un proyecto mejor, vamos a hacer una planta espacial. Y cambian el proyecto y lo ponen al año 2045». Entonces lo que nosotros decimos es que hagamos las cloacas ya, después si teniendo cloacas se les ocurrió otra cosa, que lo hagan, pero antes queremos las obras en el barrio (entrevista a Alfredo, Foro Hídrico, 12-07-2013).

\section{EL DERECHO A LA INFORMACIÓN. LA CONSTRUCCIÓN DE CONOCIMIENTOS ALTERNATIVOS ACERCA DE LA CUESTIÓN HÍDRICA}

En la literatura que registra las experiencias de los movimientos de justicia ambiental se señala que uno de los factores detonantes de la movilización es, precisamente, la negación del problema por las agencias estatales, aspecto que muchas veces incluye el ocultamiento sistemático de la información. Esta falta de reconocimiento simbólico de las víctimas, y su destitución en tanto actores que tienen derecho a saber, es, en no pocas oportunidades, lo que impulsa el reclamo por justicia. La percepción de ser tratado como «ciudadano/a de segunda categoría» funciona como un poderoso activador de la movilización social (Capek y Gilderbloom, 1992). En ese sentido, se cuestiona una retórica técnico-burocráctica y se le contrapone un lenguaje que busca humanizar las nociones que definen y nombran el problema (Capek, 1993; Di Chiro, 1998; Gibbs, 1992). 
El primer momento de la construcción de herramientas de prueba alternativas se dio a mediados de la década de 1990, cuando los vecinos empezaron a tener problemas de inundaciones por elevamiento de las napas freáticas y la empresa Aguas Argentinas negaba sistemáticamente que la solución técnica encarada por el organismo tuviera relación con el problema. Para confrontar esta definición debieron recurrir a otras fuentes de conocimiento experto, en este caso en el Instituto Nacional del Agua, quien accedió a realizar un estudio independiente. Esta investigación dio por resultado que las incorrectas decisiones técnicas de la empresa de agua tuvieron un peso decisivo en el problema de las inundaciones (INA, 2003).

Cuando consultamos a Héctor, uno de los integrantes del Foro Hídrico, nos dijo que este proceso de búsqueda de información ha sido parte de la lucha y de ganarse un espacio en la negociación con diferentes organismos estatales. En sus palabras: «Nosotros siempre fuimos buscando datos y presentando proyectos alternativos; cuando se publicó el estudio del INA, lo bueno es que era una confirmación de lo que nosotros pedíamos, nos dio como una cierta autoridad moral» (entrevista a Héctor, Foro Hídrico, 29-04-2006).

En años recientes se han concentrado en reclamos colectivos por el derecho a la salud. Aquí, la herramienta más poderosa la constituyen los relevamientos comunitarios que buscan poner en evidencia una situación que definen como «emergencia sanitaria». Para ello han trabajado conjuntamente con la organización Médicos del Mundo y la cátedra libre de ingeniería comunitaria de la Universidad de Buenos Aires para realizar diferentes estudios epidemiológicos para el diagnóstico de la situación sociosanitaria en los barrios. El objetivo es producir datos que contribuyan a mostrar los nexos entre los problemas de contaminación del agua, la falta de servicios sanitarios y la situación de salud a escala local. Un punto clave es denunciar la ausencia del estado en materia de estudios epidemiológicos, particularmente en lo que hace a los registros de historias clínicas que tomen en cuenta la relación entre los peligros ambientales y las afecciones a la salud.

En el año 2011 la organización inició, en conjunto con Médicos del Mundo, la campaña «Paremos con el genocidio silencioso de nuestro pueblo», que consistía en el diseño y la implementación de encuestas epidemiológicas en Villa Lamadrid. Los resultados de la encuesta mostraron que el 62\% de las familias sufre inundaciones en su casa o terreno, $100 \%$ no cuenta con servicio de cloacas, $34 \%$ consume agua envasada, el barrio no cuenta con servicio de recolección de residuos y el $75 \%$ de la población no cuenta con cobertura médica. Asimismo, el estudio determinó la existencia de enfermedades respiratorias, parasitosis, gastroenteritis y dermatitis (Argenpress, 24/09/2014). 
Luego de la experiencia en Villa Lamadrid, y en el marco de la misma campaña, se replicó la encuesta en cien familias del barrio 17 de Noviembre, un asentamiento localizado en un área de rellenos industriales y atravesado por efluentes contaminados. En ese relevamiento participaron una veintena de organizaciones, agrupaciones políticas, movimientos sociales, y grupos universitarios, como la Cátedra Libre de Ingeniería Comunitaria y la Cátedra Libre de Estudios Americanistas, ambos de la Universidad de Buenos (UBA). En el marco de esta campaña, se realizaron actividades de información en la vía pública donde se difundieron los resultados del relevamiento y se generaron puntos de recepción de consultas y denuncias. Esto convocó la atención de diferentes medios de prensa; en esa ocasión, las voces de los vecinos aparecieron reflejadas en las coberturas periodísticas.

\section{EL DERECHO A LA PARTICIPACIÓN EN LAS DECISIONES DE POLÍTICA PÚBLICA QUE TIENEN CONSECUENCIAS EN LA VIDA COMUNITARIA}

En la experiencia que estamos analizando es posible constatar un aspecto característico de los movimientos de justicia ambiental y que refiere a la exigencia al Estado en términos de que garantice un tratamiento justo para todos los ciudadanos. En ese sentido, se reclama por políticas públicas que tienen un papel relevante en la estructuración del problema. En ese camino, los repertorios de reclamos avanzan un paso más allá, buscando ejercer influencia en las decisiones y modos de implementación de las soluciones propuestas.

En el caso del Foro Hídrico, el aprendizaje es que los reclamos tienen que sostenerse en el tiempo mediante propuestas concretas de intervención y dado que se trata de obras de infraestructura- donde las opciones técnicas de implementación, plazos y modalidades de intervención deben quedar abiertas al escrutinio público. La movilización para la búsqueda de soluciones y la incidencia en los procesos de implementación de la política pública es un elemento central en los marcos de acción colectiva. Como señala Carla:

Cada vez que surge un problema y que lo identificamos como un problema común, buscamos soluciones comunes y luego nos movilizamos en búsqueda de ese tipo de solución. Nosotros nunca vamos a una protesta sin una propuesta superadora de lo que el gobierno propone. Es decir, siempre llevamos una solución, no es que decimos «queremos cloacas y listo»: les decimos que las queremos de esta manera y en tales plazos, con tales parámetros. Sabemos 
lo que queremos y tratamos de divulgarlo en el barrio a través de asambleas, o de ir a una escuela y hablar con los chicos, con los papás. Es un trabajo de formación, de formar conciencia, de llamar a la movilización, y después nos reunimos y tratamos, con fuerza, de imponer las ideas (entrevista a Carla, Foro Hídrico, 23-08-2014).

A partir de un análisis de las obras propuestas por el Plan Director de AySA, los vecinos del Foro Hídrico han llegado a la conclusión de que se trata de un modelo centralizado que requiere la construcción de grandes colectores y emisarios (que fueron incluidos y luego demorados o suspendidos en los planes de anteriores gobiernos) lo que supondría demorar los plazos de concreción en una o dos décadas. En contrapartida, alientan la construcción de plantas modulares que podrían brindar cobertura en corto tiempo.

Después de varias marchas terminan prometiendo cloacas para 150000 personas en la zona lindera con el barrio. Como eso es insuficiente, seguimos haciendo marchas, y la promesa ahora es no para 150000 , sino para 300000 , pero en dos etapas. Antes prometían que 160000 iba a tenerlas (60 000 de Lanús, 100000 de Lomas), digo... ¿cómo creer en todas las cuestiones que nos vienen prometiendo hace tres años? Si antes nos prometían algo y no se cumplió, ahora nos prometen esto, que bienvenido sea y que ya es por la totalidad de Lomas de Zamora. Es una buena señal que haya cambiado el Plan Director, en cuanto a lo que nosotros venimos marcando, de que no podemos esperar a su proyecto que dicen que es para el $2020 \mathrm{y}$ a eso hay que calcularse unos años más. O sea, estamos hablando de tener cloacas para el año 2025, y el costo sanitario de 600000 personas, de acá a ese momento es terrible. Entonces nosotros decimos que las plantas modulares son más rápidas, y eso lo demuestra; ellos dicen, en tres años les podemos dar cobertura (entrevista a Alfredo, Foro Hídrico, 24-02-2015).

Se trata de una forma de territorializar el proceso de implementación de las obras, logrando adelantar la ejecución sin tener que supeditar el proceso a plazos de negociación con organismos internacionales de crédito y a las diferentes pujas interestatales por la distribución de fondos para el financiamiento de las obras. Y este es un reclamo por justicia distributiva (que las obras lleguen al territorio implica compensar un déficit histórico), pero también una exigencia de reconocimiento en la que interviene la justicia procedimental. Esto implica tomar en cuenta los mecanismos de participación en términos de si se produce un cambio efectivo en el balance de las relaciones de poder en favor de los afectados.

En la experiencia de los grupos que integran el Foro Hídrico, el acceso a la toma de decisiones sobre las obras siempre ha estado en relación directa con la 
presión política mediante medidas de acción directa sobre diferentes organismos jurisdiccionales que tienen competencia en el problema. De este modo, cada movilización logra compromisos para el avance de las obras, pero luego este proceso entra en un impasse, por lo que es necesario otra vez «ganar la calle» para lograr el avance en la implementación. Como señala Alfredo, en referencia a un logro organizativo que ha sido el compromiso de una planta modular de tratamiento de efluentes cloacales en el barrio:

¿Es la planta de tratamiento de efluentes cloacales de Dock Sud la solución? El tiempo lo dirá. Es una megaplanta para miles de personas que está planificada, fundamentalmente, para la capital y la zona norte donde van los efluentes [...]. El hecho es, cortamos ahí, no dejamos llegar hasta ahí, entonces toda la capacidad de lo que venía se la damos a la zona sur. Entonces, nosotros tenemos que continuar movilizados, porque si no, no vamos a conseguir las obras (entrevista a Alfredo, Foro Hídrico, 12-07-2013).

\section{REFLEXIONES FINALES. LA CONSTRUCCIÓN SOCIAL Y POLÍTICA DE LA CUESTIÓN HÍDRICA}

En este artículo hemos analizado el proceso de definición colectiva de la cuestión hídrica en un área territorial con altos niveles de degradación ambiental. Si durante muchos años este debate había quedado invisibilizado respecto de otros reclamos sociales, en la última década esta discusión ha ganado carácter público como asunto social y político.

Si bien la definición de estos asuntos guarda relación con la constitución física del ambiente, se desarrolla fundamentalmente mediante un proceso de valoración social mediante el cual diferentes actores han podido definir y considerar cómo es que los flujos del agua son manipulados por determinadas geografías del poder (Swyngedouw, 2009). Los colectivos movilizados, al reclamar por las afectaciones ambientales y los impactos en la salud, están planteando un problema de inequidad intergeneracional y, además, abren caminos para la discusión sobre quiénes se apropian de la renta urbana en el proceso histórico de ocupación del suelo en Buenos Aires.

En términos políticos, al generar acciones públicas, campañas, movilizaciones y reclamos, estos actores han logrado hacer público el conflicto por el acceso al agua y el saneamiento, planteando que se trata de un problema de derechos conculcados. Si los movimientos de justicia ambiental exigen un tratamiento justo para todas las personas en lo que se refiere a la elaboración, desarrollo, 
implementación y aplicación de políticas, leyes y regulaciones ambientales, uno de los saldos importantes de este conflicto es que los actores han logrado posicionar públicamente esta cuestión como un asunto que merece atención estatal.

Un elemento clave del proceso ha sido la elaboración de dispositivos de exploración y de aprendizaje colectivo donde se analizan los problemas, se buscan pruebas y se pone en discusión quiénes son los actores acreditados para generar conocimiento válido. Los vecinos que integran el Foro Hídrico de Lomas de Zamora han podido realizar un aprendizaje acerca de las características del ciclo hidrosocial, un conocimiento que es altamente significativo en términos de la construcción de saberes territoriales que permiten responder, resistir, y asimismo, proponer dispositivos de implementación. Estas visiones que producen una inscripción territorial del problema se originan en modos de construcción del conocimiento que son fundamentales para gestionar la incertidumbre y que son altamente significativas para un enfoque de ciencia postnormal (Funtowicz y Ravetz, 1993).

Estas acciones colectivas cuestionan las acciones de las agencias estatales y las corporaciones en términos de una economía política del conocimiento (Sismondo, 2010), produciendo debates sobre la legitimidad de los saberes que están en juego, aspecto que puede resumirse en dos preguntas importantes: ¿cuál es el conocimiento que cuenta? y ¿quién lo produce?

Para que se pueda romper la brecha de la desigualdad en la cobertura de agua y saneamiento, es necesario que AySA pueda desplazarse de un modelo basado en la oferta. La red de agua y saneamiento se mueve desde el norte hacia el sur y desde las zonas altas hacia las bajas con la lentitud propia de una infraestructura que estuvo pensada para abastecer las zonas centrales y población con capacidad de pago. El Plan Director de Agua y Saneamiento que se propuso en 2006 demanda financiamiento externo para continuar y las previsiones indican que la inversión en los barrios de la cuenca baja del Matanza-Riachuelo podría concretarse en no menos de una década. Es por ello que la expansión de las infraestructuras de agua y saneamiento requiere la continuidad en el modelo de gestión y de un enfoque en clave de derechos, porque si se considera que el recurso hídrico es un bien de mercado, posiblemente se mantenga la brecha histórica en materia de agua y saneamiento.

Como señala Iris Young (1990), pensar que los derechos se cumplen solo porque hay igualdad de oportunidades ignora tanto la filiación de los individuos a diferentes grupos, que en una sociedad dada están desigualmente ubicados, como el desenvolvimiento histórico de las relaciones de fuerza entre esos grupos dentro 
de una sociedad. Como hemos podido ver, las organizaciones del Foro Hídrico reclaman justicia distributiva (obras de saneamiento, acceso al agua potable, gestión del riesgo), pero también ponen en cuestión la justicia procedimental, en términos de cómo se establecen las reglas para arribar a las decisiones, un aspecto directamente relacionado con las diferencias en la capacidad de influencia de los grupos sociales (Paavola, 2007, p. 96).

Los integrantes del Foro Hídrico exigen ser reconocidos y que se tomen en cuenta las prácticas representativas, interpretativas y comunicativas de la propia cultura (Fraser y Honneth, 2006). El «no reconocimiento» es un tipo de opresión que deforma y moldea la concepción que dichos seres humanos tienen de sí mismos (Taylor, 1993). Es por eso que estos actores, al igual que otros que reclaman por justicia ambiental, exigen un igual tratamiento ante la ley, lo que implica una batalla por el respeto y la autodeterminación (Harvey, 1996, p. 387; Douglas, 1984, p. 3).

Un elemento a destacar es el modo en que estos actores realizan diversos ejercicios de fiscalización de las obras de infraestructura, generando saberes para el control de gestión de las políticas públicas, lo que los convierte en portadores de un conocimiento válido que permite abrir interrogantes sobre las opciones de implementación y las decisiones de largo plazo. Son lenguajes de valoración que cuestionan enfoques tecnocráticos y traen al centro de la escena el diálogo entre saberes como prerrequisito para la participación extendida en la definición del problema.

\section{REFERENCIAS BIBLIOGRÁFICAS}

Akrich, Madeleine, Yannick Barthe y Catherine Rémy (2010). Sur la piste environnementale. Menaces sanitaires et mobilisations profanes. París: Presses des Mines. https://doi.org/10.4000/books.pressesmines.306

Acselrad, H., C. Campello do Amaral Mello y G. Das Neves Bezerra (2009). O que é justiça ambiental. Río de Janeiro: Garamond Universitaria.

Acselrad, H. (org.) (2004). Conflitos ambientais no Brasil. Río de Janeiro: RelumeDumara.

Agua y Saneamientos Argentinos S.A. (2009). «Estudio Socioeconómico en Villas y Asentamientos»; vol. I, II, III y IV.

Bernstein, M. y M. Jasper (1998). Les tireurs d'alarme dans les conflits sur les risques technologuiques. Enre intérets particuliers et crédibilité. En Politix, Revue 
des sciences sociales $d u$ politique, 44. Politiques du risque, Paris, quatrième trimester, 1988.

Blanchon, D. et al. (2009). Comprendre et construire la justice environnementale. Annales de Géographie, 1(665-666), 35-60. https://doi.org/10.3917/ag.665.0035

Brown, P. (1987). Popular epidemiology: community response to toxic waste-induced disease in Woburn, Massachusetts. Science, Tecnology, \& Human Values, 12, (3/4), 78-85.

Brown, P. y E. J. Mikkelsen (1990). No Safe Place: Toxic Waste, Leukemia, and Community Action. Berkeley: University of California Press.

Budds, Jessica y Leonith Hinojosa (2012). Restructuring and rescaling water governance in mining contexts: the coproduction of waterscapes in Peru. Water Alternatives, 5(1), 119-137.

Bullard, R. D. (1994). Dumping in Dixie: Race, Class and Environmental Quality. San Francisco/Oxford: Westview Press.

Callon, M., P. Lascoumes e Y. Barthe (2001). Agir dans un monde incertain. Essai sur la démocratie technique. París: Seuil.

Capek, S. (1993). The «Environmental Justice» Frame: A Conceptual Discussion and an Application. Social Problems, 40(1), Special Issue on Environmental Justice (Feb., 1993), 5-24 https://doi.org/10.1525/sp.1993.40.1.03x0069q

Capek S. y J. I. Gilderbloom (1992). Community Versus Commodity: Tenants and the American City. Albany, N.Y.: State University of New York Press.

Cefaì, Daniel (2007). Pourquoi se mobilise-t-on? Les théories de l'action collective. París: La Découverte-Mauss.

Catenazzi, A. (2006). Universalidad y privatización de los servicios de saneamiento. El caso de la concesión de obras sanitarias de la nación en la región metropolitana de Buenos Aires. 1993-2003. En D. Azpiazu, A. Catenazzi y K. Forcinito (eds.), Recursos públicos, negocios privados. Agua potable y saneamiento ambiental en el $A M B A$ (pp. 113-174). Serie Informe de Investigación № 19, ICO - UNGS, Los Polvorines.

Cravino, María Cristina (2008). Los mil barrios informales en el AMBA. Los Polvorines: Paidós-UNGS.

De Gouvello, B., E. Lentini y E. Brenner (2012). Changing paradigms in water and sanitation services in Argentina: towards a sustainable model? Water International, 37(2), 91-106. https://doi.org/10.1080/02508060.2012.662730

Descola, P. (2005). Par-delà la nature et culture. París: Gallimard, Bibliothèque des sciences humaines. 
Di Chiro, G. D. (1998). Nature as Community: The Convergence of Environment and Social Justice. En D. Faber (ed.), The Struggle for Environmental Democracy: Environmental Justice Movements in the United States. Nueva York: Guilford.

Douglas, M. (1984). Purity and Danger: An Analysis of Concepts of Pollution and Taboo. Londres: Ark Paperbacks [trad. esp.: Pureza y peligro. Análisis de los conceptos de contaminación y tabú. Madrid: Siglo XXI, 2000].

Faber, Daniel (2005). Building a Transnational Environmental Justice Movement: Obstacles and Opportunities in the Age of Globalization. In Joe Bandy y Jackie Smith (eds.), Coalitions Across Borders: Negotiating Difference and Unity in Transnational Struggles Against Neoliberalism. Nueva York: Roman \& Littlefield.

Firpo Porto, Marcelo (2012). Movements and the Network of Environmental Justice in Brazil. Environmental Justice, 5(2), 100-104. https://doi.org/10.1089/ env.2011.0012

Fraser, N. y A. Honneth (2006). ¿Redistribución o reconocimiento? Madrid: Morata.

Funtowicz J. y R. Ravetz (1993). Science for the postnormal age. Futures, 25(7), 739755. Pergamon.

Gibbs, L. (1992). Love Canal, My Story. Albany, N.Y.: State University of New York Press.

Guha, R. (1989). Radical American Environmentalism and Wilderness Preservation: A Third World Critique. Environmental Ethics, 11, 71-83. https://doi.org/10.5840/ enviroethics198911123

Guha, R. y J. Martínez-Alier (1997). Varieties of environmentalism. Essays North and South. Londres: Earthscan.

Harvey, D. (1996). Justice, Nature, and the Geography of Difference. Oxford: Blackwell.

Jaramillo, S. (2008). Reflexiones sobre la «informalidad» fundiaria como peculiaridad de los mercados de suelo en las ciudades de América Latina. Territorios, 18-19, Bogotá, Universidad de los Andes (pp. 11-53). Disponible en: http//redalyc.uaemex.mx

Martínez Alier, J. (2004). El ecologismo de los pobres. Conflictos ambientales y lenguajes de valoración. Barcelona: Flacso Ecología-Icaria Antrazit.

Melé, P (2007). Identifier un régime de territorialité réflexive. Colloque: Territoires, territorialité, territorialisation. Grenoble: Université Joseph Fourier, UMR Pacte Territoire, CNRS, 7 y 8 de junio.

Merlinsky, M. G (2007). Conflicto ambiental, organizaciones y territorio en el Área Metropolitana de Buenos Aires. En Andrés Solari Vicente y Anabel Cruz Santacroce (comps.), Sociedad civily desarrollo local. México D. F.: International 
Society for the Third Sector Research. Editorial Porrúa - Universidad Michoacana de San Nicolás Hidalgo.

Merlinsky, M. G. (2013). Política, derechos y justicia ambiental. El conflicto del Riachuelo. Buenos Aires: Fondo de Cultura Económica.

Merlinsky, M. G. (2014). Cartografias del conflicto ambiental en Argentina. Buenos Aires: CICCUS/CLACSO. Disponible en: http://biblioteca.clacso.edu.ar/clacso/ se/20140228033437/Cartografias.pdf

Merlinsky, G. (2016). Cartografias del conflicto ambiental en Argentina 2. Buenos Aires: Editorial CICCUS/CLACSO. Disponible en: http://www.clacso.org.ar/ libreria-latinoamericana/libro_detalle.php?orden=\&id_libro=1177\&pageNum rs_libros $=0 \&$ totalRows_rs_libros $=1121$

Merlinsky, M. G. (2016b). Mists of the Riachuelo: River Basins and Climate Change in Buenos Aires. Latin American Perspectives, 43:43-55, July 2016. https://doi. org/10.1177/0094582X15623764

Merlinsky, G., S. Fernández Bouzo, C. Montera y M. Tobías (2012). La política del agua en Buenos Aires: nuevas y viejas desigualdades. Rethinking Development and Inequality - An International Journal for Critical Perspectives, 1(1), 49-59.

Molle, François (2012). La gestion de l'eau et les apports d'une approche par la political ecology. En Denis Gautier y A. Tor Benjaminsen (dir.). L'approche Political Ecology: Pouvoir, savoir, environnement (pp. 219-240). París: Quae.

Naguib Pellow, David y Robert Brulle (eds.) (2005). Power, Justice and the Environment. A Critical Appraisal of the Environment Movement. Cambridge: MIT Press.

Paavola, J. (2007). Institutions and Environmental Governance: A Reconceptualization. EcologicalEconomics, 63,93-103.https://doi.org/10.1016/j.ecolecon.2006.09.026

Pereira, P. y M. Tobías (2014). Políticas de agua potable y saneamiento y demandas locales en el Área Metropolitana de Buenos Aires. En M. G. Merlinsky, Cartografias del conflicto ambiental en Argentina. Buenos Aires: CICCUS-CLACSO.

Schlosberg, David (2012). Justicia ambiental y climática: de la equidad al funcionamiento comunitario. Ecología Política, 41, 25-35. Barcelona: Icaria.

Sismondo, Sergio (2010). An Introduction to Science and Technology Studies. 2nd ed. Chichester: Wiley-Blackwell.

Snow, D., E. Burke Rochford, S. Worden y R. Benford (1986). Frame alignment processes, micromobilization and movement participation. American Sociological Review, 51(4), 464-481. https://doi.org/10.2307/2095581

Stake, R. (1995). The Art of Case Study Research. California: SAGE [trad. esp.: Investigación con estudios de casos. Madrid: Morata, 1998]. 
Svampa, M. (2008). Cambio de época. Movimientos sociales y poder político. Buenos Aires: Siglo Veintiuno Editores.

Swyngedouw, E., M. Kaïka y E. Castro (2002). Urban water: A political-ecology perspective. Built Environment, 28(2), 124-137.

Swyngedouw, Erik (2009). The Political Economy and Political Ecology of the HydroSocial Cycle. Journal of Contemporary Water Research \& Education, 142(1), 56-60. https://doi.org/10.1111/j.1936-704X.2009.00054.x

Swyngedouw, Erik (2004). Social Power and the Urbanization of Water: Flows of Power. Oxford: Oxford University Press.

Tobías, M. (2014). La re-estatización del servicio de agua y saneamiento y la gobernabilidad del agua en el Área Metropolitana de Buenos Aires (2006-2012) (tesis de maestría no publicada). Instituto de Altos Estudios Sociales - Universidad Nacional de San Martín.

Tobías, M. (2016). El acceso al agua en Buenos Aires durante la era post-neoliberal: ¿Derecho humano o commodity? En Gabriela Merlinsky (comp.), Cartografias del conflicto ambiental en Argentina II (pp. 259-286). Buenos Aires: Ediciones CICCUS/CLACSO.

Taylor, Ch. (1993). El multiculturalismo y la «política del reconocimiento». México D.F.: Fondo de Cultura Económica.

Trom, D (1999). De la Réfutation de L'Effet Nimby considérée comme une pratique militante. Notes pour une approche pragmatique de l'activité revendicative. Revue Française de science politique, 49, 31- 50. París; Presses de Sciences Po.

Vainer, Carlos (2004). Águas para a vida, não para a morte. Notas para uma história do movimento de atingidos por barragens no Brasil. En Henri Acselard et al. (comps.), Justiça ambiental e cidadania (pp. 185-215). Rio de Janeiro: Relume Dumara.

Young, I. M. (1990). Justice and the Politics of Difference. Princeton: Princeton University Press.

\section{FUENTES CONSULTADAS}

Periódico Punto Cero. Nota reunión del Foro Hídrico con autoridades. https:// puntocero2006.wordpress.com/2006/09/20/lomas-de-zamora-el-foro-hidricose-reunio-con-autoridades-provinciales-en-la-plata-por-la-crisis-en-la-region/. (Consultado el 16-02-2017).

El agua no es confort. Informe de la Campaña realizada por el Foro Hídrico y la Cátedra de Ingeniería Comunitaria de la UBA. Disponible en: https:/es.scribd.com/ document/260050083/2015-Informe-Foro-Hidrico-Causa-Riachuelo. 